eine Diskussion über den Begriff „Katholische Aufklärung“ zu führen. Hinsichtlich der Apodiktik, mit der in mehreren Aufsätzen behauptet wird, es habe eine Katholische Aufklärung gegeben, und hinsichtlich des hier und da offensichtlich apologetischen Gebrauchs dieses Terminus, wird der Sammelband vielleicht sogar Zweifel bei Forscherinnen und Forschern säen, die - wie der Rezensent - die These, es habe eine Katholische Aufklärung gegeben, mit Wohlwollen betrachten.

Funding Open Access funding enabled and organized by Projekt DEAL.

Open Access Dieser Artikel wird unter der Creative Commons Namensnennung 4.0 International Lizenz veröffentlicht, welche die Nutzung, Vervielfältigung, Bearbeitung, Verbreitung und Wiedergabe in jeglichem Medium und Format erlaubt, sofern Sie den/die ursprünglichen Autor(en) und die Quelle ordnungsgemäß nennen, einen Link zur Creative Commons Lizenz beifügen und angeben, ob Änderungen vorgenommen wurden.

Die in diesem Artikel enthaltenen Bilder und sonstiges Drittmaterial unterliegen ebenfalls der genannten Creative Commons Lizenz, sofern sich aus der Abbildungslegende nichts anderes ergibt. Sofern das betreffende Material nicht unter der genannten Creative Commons Lizenz steht und die betreffende Handlung nicht nach gesetzlichen Vorschriften erlaubt ist, ist für die oben aufgeführten Weiterverwendungen des Materials die Einwilligung des jeweiligen Rechteinhabers einzuholen.

Weitere Details zur Lizenz entnehmen Sie bitte der Lizenzinformation auf http://creativecommons.org/ licenses/by/4.0/deed.de.

\title{
Ritschel, Gregor: Jeremy Bentham und Karl Marx. Zwei Perspektiven der Demokratie, 360 S., transcript, Bielefeld 2018.
}

\author{
Skadi Siiri Krause
}

Online publiziert: 15 . Juli 2020

(C) Der/die Autor(en) 2020

Jeremy Benthams Ablehnung des Common Law, für ihn verkörpert in der Arbeit des damals hochgeachteten William Blackstone, ist bekannt. Nach den revolutionären Ereignissen in Amerika und Frankreich kritisierte er öffentlich die Vorstellung eines Gesellschaftsvertrags, die Behauptung amerikanischer und französischer Revolutionäre, die Menschen seien mit Naturrechten ausgestattet, die Verletzung von Men-

\footnotetext{
S. S. Krause $(\bowtie)$

Technische Universität Darmstadt, Darmstadt, Deutschland

E-Mail: skadi.krause@tu-darmstadt.de
} 
schenrechten mache positives Recht ungültig und rechtfertige zivilen Ungehorsam. All dies machte ihn in den Augen seiner Zeitgenossen nicht gerade zu einem Avantgardisten. Und doch stand er für das Ringen um eine neue politische Wissenschaft, die sich allgemeingültigen Methoden und Maßstäben verpflichtete. Deshalb war es für ihn wichtig, die dem Recht und Rechtsdenken zugrundeliegenden gesellschaftlichen Interessen aufzudecken. Nicht zuletzt in diesem Punkt hatte Benthams Ansatz Ähnlichkeiten mit der marxistischen Kritik an der Hegemonie.

Gleichwohl mag der Vergleich beider Denker überraschen, hatte Karl Marx gegenüber Bentham doch meist nur sarkastische Äußerungen parat: ,Jeremias Bentham ist ein rein englisches Phänomen. [...] Wenn ich die Courage meines Freundes H. Heine hätte, würde ich Herrn Jeremias ein Genie in der bürgerlichen Dummheit nennen“ (MEGA II/6, S. 558f.). Mit diesen Worten wandte Marx den Vorwurf Benthams der interessengeleiteten Theorien auf diesen selbst an. Das wirft die Frage auf, warum sich der Vergleich der beiden Autoren, deren Gesamtwerk sich in der inhaltlichen Ausrichtung deutlich unterscheidet und die nicht einmal im werkgeschichtlichen Sinne Zeitgenossen waren (Bentham publizierte hauptsächlich in der zweiten Hälfte des 18. Jahrhunderts, Marx in der zweiten Hälfte des 19. Jahrhunderts), dennoch lohnt.

Die Antwort, die Gregor Ritschel in seiner detailreich ausgearbeiteten Monografie gibt, bleibt, um es gleich vorwegzunehmen, unbefriedigend. So will er zum einen aufzeigen, dass sich Marx utilitaristischer Argumente bediente und seine Vorstellung vom Kommunismus am Prinzip ,,des größten Glücks der größten Zahl“ orientiert war (S. 11). Dafür hätte es allerdings nicht der Gegenüberstellung beider Denker und schon gar nicht einer monografischen Arbeit bedurft. Zum anderen will Ritschel nachweisen, und hier liegt der eigentliche Kern des Problems, dass beide Denker gleiche Ziele verfolgten: „Beide wollten erstarrte Ungleichgewichte, die Minderheiten zugunsten der Mehrheiten privilegieren, aufbrechen und zudem nach Wegen zur gleichberechtigten Freiheit aller Individuen suchen“(ebd.). Eine solche These zu entwickeln, würde jedoch beinhalten, werkgeschichtliche Stränge herauszuarbeiten und die Kritik der beiden Denker in den politischen wie wissenschaftlichen Kontext einzuordnen. Doch eben diesen umfassenden Nachweis bleibt Ritschel schuldig.

Was ihm allerdings durch die Gegenüberstellung der beiden Denker gelingt, ist, die tradierten rezeptionsbedingten Gegensätze zwischen dem ,,marktliberalen“ Bentham und dem ,illiberalen“ Marx, die er ausführlich in den ersten beiden Kapiteln seiner Monografie darlegt, aufzubrechen. Gleichwohl bleibt die Einordnung von Bentham und Marx als Demokratietheoretiker beziehungsweise Kritiker ,im Namen der Demokratie“ mehr als fraglich (S. 67). Um eine solche These aufstellen zu können, wäre es notwendig, das demokratische Denken im 18. und 19. Jahrhundert - sowohl in England als auch auf dem Kontinent - darzulegen, Denkschulen herauszuarbeiten und voneinander abzugrenzen, und Weiterentwicklungen zu benennen. Dies verabsäumt Ritschel aber in dem dafür prädestinierten dritten Kapitel, indem er Marx' und Engels' frühe Rezeption Benthams thematisiert. Dieses wäre auch dafür geeignet gewesen, das Politikverständnis der beiden Denker stärker herauszuarbeiten und abzugrenzen. Ritschel kommt dem insofern nach, als er herausstellt, dass Bentham sich als Pionier verstand, der sich auf unterschiedlichen Gebieten für Sozialreformen unter dem rechtsphilosophischen Grundsatz des „Glücks der größ- 
ten Zahl“" einsetzte. Marx hingegen betrachtete die Gesellschaft als tief gespalten und versuchte auf Grundlage einer ökonomischen Analyse sozio-politische Umwälzungen zu prognostizieren und zu rechtfertigen. Was das für das Politik- und Demokratieverständnis beider Denker bedeutete, führt Ritschel allerdings nicht näher aus.

Wie unterschiedlich die beiden Denker politisch agierten, verdeutlicht Ritschel sehr gut im vierten Kapitel seiner Arbeit, in dem er ihre Konzepte von Staat, Recht und Öffentlichkeit, aber auch ihre Ansichten zu Handlungsfeldern wie soziale Fürsorge und Kolonialpolitik gegenüberstellt. Gerade dieses Kapitel macht jedoch ebenso deutlich, dass die These des Buches, wonach Bentham und Marx in gleicher Weise „von einem demokratischen Grundgedanken“ (S. 327) getrieben waren, deren unterschiedliche Politikansätze vermischt. Bentham wollte die Gesellschaft reformieren, blieb dabei aber ein Liberaler, der dem Grundsatz des Rechtsstaates verpflichtet war. Marx hingegen waren Reformen nicht genug. Er beschrieb das Proletariat als eine Kraft, die die bürgerliche Gesellschaft und den liberalen Rechtsstaat letztendlich aus den Angeln heben werde. Damit ist zwar Ritschels These von den zwei Perspektiven der Demokratie, die bereits im Untertitel des Buches auftaucht, noch nicht widerlegt, fraglich bleibt aber, ob Bentham und Marx unter Demokratie und einer Demokratisierung der Gesellschaft überhaupt das Gleiche verstanden.

Funding Open Access funding provided by Projekt DEAL.

Open Access Dieser Artikel wird unter der Creative Commons Namensnennung 4.0 International Lizenz veröffentlicht, welche die Nutzung, Vervielfältigung, Bearbeitung, Verbreitung und Wiedergabe in jeglichem Medium und Format erlaubt, sofern Sie den/die ursprünglichen Autor(en) und die Quelle ordnungsgemäß nennen, einen Link zur Creative Commons Lizenz beifügen und angeben, ob Änderungen vorgenommen wurden.

Die in diesem Artikel enthaltenen Bilder und sonstiges Drittmaterial unterliegen ebenfalls der genannten Creative Commons Lizenz, sofern sich aus der Abbildungslegende nichts anderes ergibt. Sofern das betreffende Material nicht unter der genannten Creative Commons Lizenz steht und die betreffende Handlung nicht nach gesetzlichen Vorschriften erlaubt ist, ist für die oben aufgeführten Weiterverwendungen des Materials die Einwilligung des jeweiligen Rechteinhabers einzuholen.

Weitere Details zur Lizenz entnehmen Sie bitte der Lizenzinformation auf http://creativecommons.org/ licenses/by/4.0/deed.de. 